\title{
The role of letters in word identification: A test of the pattern-unit model
}

\author{
NEAL F. JOHNSON \\ Ohio State University, Columbus, Ohio 43210
}

\begin{abstract}
Three experiments are reported in which subjects were asked to read short stories and search for either a word with a particular meaning or a word that began with a particular letter. The results of the experiments were consistent with prior data in demonstrating that subjects were able to make word-level decisions faster than they could make component-level decisions. In addition, the final experiment indicated that subjects were no faster at finding a specific predesignated target word than they were at finding a word that belonged to a predesignated target category. The results were interpreted as supporting a model of pattern perception that assumes that the cognitive encoding of a small pattern does not entail any analogous, but prior, encoding of its components. In addition, the final experiment might suggest that the word-level encoding contains a semantic component.
\end{abstract}

During the past few years, there has been an increasing interest in the nature of the part-whole relationships that might be involved in the perception of small patterns. In particular, interest has focused on the role played by component information when a subject's task is either to identify or to classify a small pattern, and much of the research has seemed to center on the question of whether such a pattern-level identification can occur prior to a more or less complete identification of the pattern's components. In addition, while there has been some work that has involved pictures (Biederman, 1972) and other relatively complex patterns (Navon, 1977; Palmer, 1977), the bulk of the research has been on the role of letters in word identification. Specifically, a great deal of work has dealt with the question of whether word identification must be preceded by an analogous identification of the word's component letters.

One experimental paradigm that has been used to examine this issue involves determining whether the time needed to respond to a word is greater or less than the time needed to respond to a letter within a word (Johnson, 1975). If subjects do base word identification on a prior identification of the word's component letters, then the time needed to determine that a displayed word conforms to a predesignated target word should be longer than the time needed to determine that the displayed word contains a predesignated target letter.

While that expectation seems quite clear, the data have not supported the type of bottom-up view of word processing implied by this position (LaBerge, 1976). In fact, the results of a variety of experiments indicate just the reverse to be the case (Johnson, 1975; Marmurek, 1977; Sloboda, 1976, 1977), in that subjects seem able to make word-level decisions substantially faster than they can make component-level decisions. Slofoda (1977) has labeled this experimental outcome the word-priority effect, and it does seem to suggest that word patterns might have a tendency to conceal their component letters.

\section{Interpretations of the Word-Priority Effect}

There have been at least three different attempts to deal with these data, and each approach differs from the others in important ways. The original interpretation was in terms of the pattern-unit model (Johnson, 1975, 1977). That view is based on the central assumption that whenever a small pattern is to be processed into memory, the initial attempt always is to assign a single unitary encoding to the entire array, without any preliminary cognitive encoding of the components. If that step is unsuccessful, as might be the case if the display is an unfamiliar string of consonants, then it is necessary for the subject to parse the array and process it into memory by assigning a separate code to each of its components. Under those circumstances, the most immediately available information would be encodings of the individual components, and if the array had to be compared with a predesignated target array, the comparison could be done only by way of a set of componentlevel matches. In this case, then, the component-level information would be more immediately available to the subject than would word-level information.

The situation would be somewhat different if the display could be assigned a unitary encoding, as would be the case if it was an orthographically regular word. Under those circumstances, the cognitive representation most immediately available to the subject would be the unitary word-level encoding that had been assigned to the display. If the subject's task was to compare that encoding with a similar encoding for a predesignated target word, it could be done quite quickly and without any further processing. However, if the subject's task was to determine whether the word display contained a 
predesignated target letter, it then would be necessary to decode the word-level code into its components before determining whether any element matched the encoding of the target. That extra decoding step could account for the delay in responding in that situation.

A second interpretation of the data has been noted by Henderson (1975). He pointed out that when a display did not conform to the target (i.e., it was a foil or "no" item), it also was the case that the two items rarely if ever shared any letters. Given that situation, there was more letter information differentiating targets and foils when the targets were words than when they were letters within the words, and the differential richness of the stimulus information in the two tasks might have resulted in slight criterion changes that could account for the data.

For example, if subjects did identify letters before identifying words, the absence of an overlap in letters between targets and foils would allow the subjects to truncate the entire word identification process and to respond on the basis of the first letter that was encoded. If that letter was consistent with the target word, the response almost certainly would be "yes," whereas it would have to be "no" if the first encoded letter was inconsistent with the target. On the other hand, if the subject had to respond to a particular letter within the word, the process would take somewhat longer, because that letter might not be the first one that was encoded. In fact, in the case of a foil display, all the letters would have to be encoded into memory before the subject could respond accurately.

While it is clear that this view can handle the wordpriority effect when targets and foils do not share letters, more recent studies have demonstrated that the effect also can be obtained when the problem of targetto-foil similarity is controlled (Sloboda, 1976, 1977; Johnson \& Pettegrew, Note 1). For example, if the predesignated target was either the letter $\mathrm{N}$ or the word "nest," the positive display would be NEST, while a foil display might be BEST. Clearly, response determination in this case would have to be based on exactly the same stimulus information, regardless of the nature of the target, and yet even under these circumstances, a reaction time advantage was obtained for word targets over targets that were letters within words.

The third interpretation of the data has been provided by Massaro and Klitzke (1977). They began by assuming that as an initial step in the processing of words, subjects extract feature information from all the letter positions within the word in parallel. However, if the targets and foils rarely shared letters, this view also would suggest that the processing might be truncated. In particular, the subject could respond immediately after the first feature had been extracted from each letter position, because if they were all consistent with the target, the likelihood of a match would be very high, while even a single inconsistent feature would signal a mismatch. On the other hand, if the subject's task was to respond to a letter within the word, the fact that many letters share the same features would require subjects to extract many features from each letter position before they could be certain of the correct response. That extra feature extraction could result in a longer reaction time when subjects respond to letters within words than when they respond to words.

Again, however, the outcomes of more recent experiments have not been completely consistent with this interpretation. The previously described data by Sloboda (1976, 1977) and Johnson and Pettegrew (Note 1) would seem to be particularly troublesome for this position, in that both letter-and word-level decisions would require exactly the same feature information. In addition, while this view would predict that subjects need fewer features per letter position to make word-level decisions than to make letter-level decisions, Cattell (1886), Johnson (1975), and Marmurek (1977) all have demonstrated that subjects cannot respond more efficiently to words than to letters in isolation.

Massaro and Klitzke (1977) did attempt to deal with this latter problem by arguing that while word-level decisions would have the advantage of requiring fewer features per letter position, that advantage might be offset by a slower process of feature extraction for words as a result of lateral inhibition. If that were the case, they suggest that if that advantage for words was eliminated by having targets and foils with overlapping letters, then only the negative effect of lateral inhibition would remain, and words should have longer reaction times than letters in isolation.

The results reported by Massaro and Klitzke (1977) did support their prediction in this situation, but it should be noted that their demonstration does not address the most important consideration in this issue. That is, the critical issue is not whether target-to-foil similarity might delay word-level decisions in comparison to decisions regarding letters in isolation, but, rather, whether such similarity eliminates the advantage of word-level decisions over decisions regarding letters within words. While there may be a number of variables that could delay word-level decisions, the real question is whether those variables also would cause an equal delay in the subject's response to letters within words. The Sloboda $(1976,1977)$ and Johnson and Pettegrew (Note 1) studies would indicate that to be the case.

\section{A Reevaluation of the Issue}

One of the difficulties involved in attempting to resolve the problem of part-whole relationships in pattern perception is that the conceptual basis of the issue is not completely unambiguous. For example, while it is tempting to question whether words can be identified prior to an analogous identification of their 
component letters, it also is abundantly clear that subjects are unlikely ever to make a mistake in identifying or making a discrimination between items such as "lend" and "send." The fact that subjects have that skill suggests that the letter information must be available to them in some form prior to a viable word-level representation. Similarly, "show" and "snow" differ only in terms of whether the middle of the second letter is occupied by a diagonal or a horizontal line, and that feature information also must be available to the reader in some form in order to avoid an encoding error. Given these considerations, then, it seems clear that if the critical question is whether letter or feature information is used in word perception, we need go no further, because these illustrations document the fact that component information is needed to detect, recognize, and identify word-level patterns.

However, as the statements above would indicate, the real question may not be whether the component information is available and used, but rather, what is the form in which it is available? As a beginning, it can be noted that when a subject identifies a word, he must have activated, and have available, some viable memory code for the word in order to select and execute the appropriate identification response. At the simplest level, it seems reasonable to ask whether the same type of memorial encoding is activated and available for the component information prior to (or at the same time as) the availability of the word-level memory code. This question becomes of interest because models such as that of Massaro and Klitzke (1977) assume component information can be used to select and make decisions about pattern-level codes.

One way to deal with this issue is to draw a distinction between a perceptual encoding of information and a cognitive or memorial representation of the same information (Johnson, 1975, 1977). While the former is assumed to be a precognitive and uninterpreted representation of the physical characteristics of the display, a cognitive-memorial encoding is assumed to be an interpreted representation that is assigned to the perceptual encoding and can be used as the basis for making response decisions.

In that it is assumed that cognitive-memorial representations are encodings that are assigned to perceptual representations, this view would expect that a perceptual encoding would have to precede a cognitive encoding of the same information. In addition, in that the feature and letter (i.e., feature arrangement) information represents the physical characteristics of the display, it is clear that this view also would expect that word processing would begin with a perceptual encoding of the feature and letter information. Therefore, given this construction of the issue, this view of word processing would expect some type of perceptual encoding of the component information prior to a viable word-level encoding, but those encodings may not be of the same form as the final word-level encoding, and they may not be useful as a basis for response selection. Within these terms, one way of restating the holistic processing hypothesis of the pattern-unit model is to say that while there may be perceptual encodings of component information prior to a unitary cognitive encoding of a word, there is no cognitive encoding of the component information that could be used for making a response decision.

\section{EXPERIMENT 1}

In light of these considerations, the purpose of the first experiment was to ascertain whether subjects could make a response decision regarding a word-level representation as fast or faster than they could make a similar decision regarding component-letter information when the difficulties of the earlier studies were either eliminated or controlled. For example, while the letter-search task in this experiment was similar to that used in the past (i.e., subjects were to find words that began with a particular letter), the word-search task was changed from having subjects search for a particular word to having them search for a word that fit a particular semantic category (e.g., the name of an animal or a flower). Under these circumstances, the subjects would not have the type of prior knowledge regarding the specific visual characteristics of the target words that is required by the Henderson (1975) and Massaro and Klitzke (1977) truncated processing hypotheses.

A second change in the nature of the task was to ask subjects to search for the targets as they were reading a short story, rather than to have them respond to single words presented in isolation. It was hoped that this change would result in a situation with enough similarity to a normal reading task to warrant the possibility of at least some generalization.

\section{Method}

The basic material consisted of a set of 24 short stories that varied in length from about 55 to about 70 words, and under normal circumstances, it took about 10-15 sec to read them. The stories were typed (double spaced) on white paper, and the lines were approximately $4.5 \mathrm{in} .(10.4 \mathrm{~cm})$ long, with each story occupying about six lines. The following is a typical story.

"The girl was lying in the sun, although the large maple started to throw a shadow. It was getting late and there was only a little time left. She would go in when the shadow got to her blanket. It would soon be time for her date to arrive and she wanted to be ready when he got there. In addition to that, her Coke was all gone."

Three target items were included within each story, with one of them appearing early, one in the middle, and one of them late in the story. These items are in italics in the illustration, but that was not the case for the displays presented to the subjects. The target items were selected such that each represented a clear instance of an easily describable category, and the first letter of the word had not appeared earlier in the story. The category descriptions used for the above illustration were "a tree name," "a common item found on a bed," and "a beverage." 
In that each story had three target items (i.e., early, middle, and late) that could be designated to the subject by either the first letter of the word (letter-search condition) or the semantic category of the word (semantic-search condition), there were actually six targets for every story. However, any one subject searched for only a single target as he read each story. Each story was used for the letter-search condition for half the subjects, and for the other half of the subjects, it was used for the semantic-search condition. In addition, within each of the halves, one-third of the subjects had the early item, one-third had the middle item, and one-third had the late item as the target.

Each subject was presented 12 stories in each of the two search conditions. For three of the stories in each condition, the target item did not appear. These were the "no" items, or foils. They were constructed by deleting the three target words from three stories and replacing them with words that: (1) were from different semantic categories; (2) did not contain the initial letter of any of the deleted targets; and (3) were consistent with the story. In each case, the predesignated targets were the original items, and in that way it was assured that the targets used for the foil items were as thematically consistent with the story as was the case for the "yes" items. Of the remaining nine stories in each condition, the early, middle, and late targets were used three times each. Finally, the order of the stories within a condition was random, but half the subjects had the letter-search task followed by the semantic-search task, and the reverse was the case for the other half. In general, then, each subject experienced each of the six conditions equally of ten, each story appeared in each condition equally often, and all these conditions were appropriately counterbalanced across subjects.

Procedure. Immediately before testing the subjects in each of the two conditions, the experimenter gave them three practice stories in which they were to search for the type of target to be used in that condition. The target appeared in two of the stories and it did not appear in one of them (i.e., it was a foil item). That practice was immediately followed, without a break, by the 12 experimental stories. For both the practice and the experimental stories, the subjects' instructions were to read the stories to themseives and to search for the target as they were reading. In addition, they were told that it was important to understand and comprehend the story, because they were going to be tested for their memory of the material after all of the stories had been presented. This part of the instruction was emphasized by repeating it more than once.

The subjects were told to read each story, searching for the target, and if they found it they were to say "yes," report the word, and then continue reading the story and say the word "done" when they had finished. In the event the story did not contain the target, they were to say "done" when they finished the story and then report that they were unable to find the target.

Finally, after reading all 24 stories, the subjects were given a 72-item comprehension test that consisted of three questions from each story, and the questions were selected to deal with issues not specifically tied to the target items. For example, for the above illustration, the three questions were the following: (1) Where was the girl lying? (2) When was she going to go in? (3) Who would be arriving? The scoring was quite liberal: Any response indicating that the subject knew the critical information was accepted. For example, for the above questions, anything related to being in the sun or getting a suntan would be accepted for the first question; anything related to the shadow hitting either the girl or the blanket would be accepted for the second question; and "date" or "boyfriend" would be equally acceptable for the third question.

When a story was presented to a subject, it was covered by a sheet of thick white paper, and the subject was then informed of the target for which he was to search. Two stopclocks were started as the cover sheet was removed. The first clock was stopped when the subject said "yes," indicating he had found the target. The second clock was stopped when the subject said "done." The experimenter then recorded both the subject's response and the two latencies.

Subjects. The subjects were 36 introductory psychology students who participated as part of a course option.

\section{Results}

The results of the experiment are presented in Table 1, with the error frequency and reaction time shown for each condition. There was a total of 14 errors in the experiment $(1.6 \%)$, with one subject making 3 errors, two subjects making 2 , and seven subjects making 1 error each. In addition, the errors were about equally distributed between the two different target-search conditions ( 6 vs. 8 ), with the slight difference going in the same direction as the difference in reaction times. All of the errors involved instances of failing to detect a target (i.e., they were all misses).

An analysis of the reaction times in Table 1 indicate that the effect of the position of the target in the story was significant $[F(2,70)=423.31, p<.001]$, as was the effect of type of target (i.e., first letter vs. semantic category) $[F(1,35)=4.43, p<.05]$, but the interaction was not significant $(\mathrm{F}<1.00)$. The time subjects needed to report that they had finished reading the story, when they had previously reported identifying the target, was $12.24 \mathrm{sec}$ for the letter-search task and $11.76 \mathrm{sec}$ for the semantic-search task $[F(1,35)=7.99, p<.01]$. Finally, for the cases in which the target did not appear in the story, the time taken by subjects to report that they had finished reading was $11.86 \mathrm{sec}$ for the letter-search task and $10.68 \mathrm{sec}$ for the semantic-search task $[F(1,35)=$ $15.67, \mathrm{p}<.01]$.

Quite clearly, it took subjects longer to find a target word in a short story when they were looking for a word that had a particular first letter than when they were looking for a word with a particular meaning. In addition, the advantage for word-level targets seemed to appear regardless of whether one considers the time to find a target, the time to completely read the story (given the target appeared in the story), or the time to determine that the target did not appear in the story.

The final issue concerns the information the subjects retained once they had finished the reading task. In terms of the final comprehension test, the maximum possible score was 36 for each condition, and the score for the letter-search condition was 12.44 while it was

Table 1

Latency Results (in Seconds) from Experiment 1

\begin{tabular}{|c|c|c|c|c|c|c|c|c|}
\hline \multirow{3}{*}{$\begin{array}{l}\text { Type of } \\
\text { Search }\end{array}$} & \multicolumn{6}{|c|}{ Target Position } & & \\
\hline & \multicolumn{2}{|c|}{ Early } & \multicolumn{2}{|c|}{ Middle } & \multicolumn{2}{|c|}{ Late } & \multicolumn{2}{|c|}{ Mean } \\
\hline & RT & $\mathrm{EF}$ & RT & $\mathrm{EF}$ & RT & $\mathrm{EF}$ & RT & $\mathrm{EF}$ \\
\hline Letter & 1.84 & 5 & 6.85 & 2 & 10.56 & 1 & 6.42 & 8 \\
\hline Semantic & 1.79 & 2 & 6.44 & 2 & 10.18 & 2 & 6.13 & 6 \\
\hline Mean & 1.82 & 7 & 6.64 & 4 & 10.37 & 3 & 6.28 & 14 \\
\hline
\end{tabular}

Note $-R T=$ reaction time, $E F=$ error frequency. 
14.08 for the semantic-search condition $[F(1,36)=3.04$, $\mathrm{p}>.05]$. It seems quite clear that the reaction time advantage for the semantic-search task did not occur at the expense of the type of processing involved in comprehension, although the comprehension scores were not very high for either condition.

\section{Discussion}

The results of the first experiment seemed to be in general accord with the expectations, as well as the prior data (Johnson, 1975; Sloboda, 1976, 1977). The subjects took longer to identify a word when using its first letter as the basis for search than when using its meaning. In addition, there was substantial evidence that the subjects were processing the stories serially from beginning to end, in that there was a marked increase in reaction time as the target appeared later in the story. In general, then, it would appear that as the subjects moved through the stories an encoding of the meaning of the words they encountered was more immediately available to them for determining a response than were encodings of the first letters of the words.

One disquieting result was the failure to obtain an interaction between the type of target and the position of the target item within the story. If the main effect of target type occurred because of a difference in the way the words were processed as they were being read, then one would expect that the difference in reaction time between the conditions should become increasingly magnified from the beginning of the story to the end. While the differences in mean reaction time did evidence a slight increase from the beginning to the end, the effect was not reliable $(F<1.00)$, and it completely disappeared in a subsequent replication.

One possible explanation for the failure to obtain an interaction could lie in the fact that the subjects reported a tendency to scan the text for the target, rather than reading carefully for comprehension. When questioned after being tested, the subjects frequently said either that they had forgotten that the comprehension test would be coming at the end of the session, or that they were under the impression that the search task was the important part of the study and that it was in that activity that they should concentrate their effort. Furthermore, that possibility is supported by the comprehension data. While the two search tasks did not result in different levels of comprehension, the overall level of comprehension was not very high for either task (37\%).

\section{EXPERIMENT 2}

It is not at all clear how the type of scanning activity described by the subjects could account for the data, but their responses in the postexperimental interviews strongly suggested that they had engaged in some type of activity that was not the same as that involved when they read for comprehension. In addition, they seemed to show genuine surprise and chagrin when they realized how poorly they had performed on the comprehension test. For that reason, it was decided to repeat the experiment, but with an extensive practice session added to the beginning of the task that was followed by a comprehension test. It was hoped that if the subjects understood the importance and difficulty of the comprehension test before they began the experimental task, they would pay more attention to that aspect of their performance.

\section{Method}

The methods, materials, and procedures for the second experiment were identical to those used in the first experiment, with the exceptions that: (1) a 14-story practice session was added to the beginning of the subjects' task; (2) the instructions placed a greater emphasis on comprehension and retention; and (3) the sample size was increased from 36 to 48 subjects. The need for this latter change was indicated by pilot data that showed that the practice session had a slight tendency to attenuate the magnitude of all of the effects obtained in the first study.

For the practice session, there were 14 new stories constructed that had the same general characteristics as the 24 stories used in the experimental session. However, since the only function of these stories was for practice, seven of them were predesignated for semantic search and the other seven were used for letter-search practice. In each practice condition, two stories had an early target, two had a middle target, two had a late target, and one was a "no" item in which a target did not appear. The order in which the two search conditions were presented to the subjects during practice was the same as during the experimental session, and after the subjects had gone through all the practice stories in both conditions they were given the comprehension test. The comprehension test consisted of 42 questions ( 3 per story), and it was similar to the one used for the experimental stories.

As expected, all of the subjects made a number of errors on the practice comprehension test, and they were informed of that fact immediately after they had finished the test. In addition, they were told that we had hoped that they would have been able to do somewhat better, because our interpretation of the experiment depended upon a high level of comprehension and retention.

At that point the subjects were told: (1) that the second part of the experiment would begin; (2) that this part would be essentially the same as the first part; and (3) that on this part of the task, they should place a greater emphasis on comprehending and retaining the stories, because there would be a critical test of their knowledge at the end of the session. From then to the end of the experimental session, everything was exactly as it had been for the first experiment, including the three practice stories that preceded the presentation of the stories in each condition.

\section{Results}

The results of the experiment are presented in Table 2. All errors were failures to detect a target, and

Table 2

Latency Results (in Seconds) from Experiment 2

\begin{tabular}{|c|c|c|c|c|c|c|c|c|}
\hline \multirow{3}{*}{$\begin{array}{l}\text { Type of } \\
\text { Search }\end{array}$} & \multicolumn{6}{|c|}{ Target Position } & & \\
\hline & \multicolumn{2}{|c|}{ Early } & \multicolumn{2}{|c|}{ Middle } & \multicolumn{2}{|c|}{ Late } & \multicolumn{2}{|c|}{ Mean } \\
\hline & RT & EF & RT & EF & RT & EF & RT & EF \\
\hline $\begin{array}{l}\text { Letter } \\
\text { Semantic }\end{array}$ & $\begin{array}{l}1.90 \\
1.98\end{array}$ & $\begin{array}{l}7 \\
3\end{array}$ & $\begin{array}{l}8.43 \\
8.02\end{array}$ & $\begin{array}{l}8 \\
5\end{array}$ & $\begin{array}{l}13.05 \\
11.75\end{array}$ & $\begin{array}{l}7 \\
9\end{array}$ & $\begin{array}{l}7.78 \\
7.25\end{array}$ & $\begin{array}{l}22 \\
17\end{array}$ \\
\hline Mean & 1.94 & 10 & 8.23 & 13 & 12.33 & 16 & 7.52 & 39 \\
\hline
\end{tabular}

Note $-R T=$ reaction time,$E F=$ error frequency. 
again, there appeared to be little difference in errors between the two search conditions (i.e., 17 vs. 22 errors). The slight difference that did occur was positively related to the reaction time data. The overall error rate was somewhat higher in this experiment than in the first $(1.6 \%$ vs. $2.6 \%)$, and whereas the errors were confined to $28 \%$ of the subjects in Experiment 1 , $63 \%$ of the subjects in Experiment 2 made an error. Finally, 1 subject made three errors, 7 subjects made two errors, and 22 subjects made one error each.

An analysis of variance of the error data indicated that none of these effects was reliable $(F<1.00)$. Therefore, while the overall error rate seemed to be somewhat higher in this experiment than in the first, the relative distribution across subjects approximated that obtained in the first experiment, and the rate was about the same for the two target-search conditions $(\mathrm{F}<1.00)$.

The mean comprehension score for the semanticsearch condition was 18.52 items, and it was 17.29 for the letter-search condition $[F(1,47)=1.07, p>.05]$. The results, therefore, indicate little or no difference in comprehension and retention between the conditions, but the overall level of performance did seem to be about $25 \%$ higher than that obtained in the first experiment.

An analysis of the latency data in Table 2 indicated significant effects of type of target $[F(1,47)=6.20$, $\mathrm{p}<.05]$ and position of the target item within the story $[\mathrm{F}(2,94)=507.32, \mathrm{p}<.001]$, but most critically, the interaction between target type and target position also was significant $[F(2,94)=3.99, p<.05]$. Given the linear regularity of the effect of position for both search conditions, the interaction would suggest that there was a reliable increase in the reaction time difference correlated with target position.

The mean time for the subjects to report that they had finished reading the story, given that they had found a target, was $14.39 \mathrm{sec}$ for the semantic-search condition and $15.39 \mathrm{sec}$ for the letter-search condition $[\mathrm{F}(1,47)=$ $20.54, \mathrm{p}<.01]$. When the target item had not appeared in the story, the time to determine that it was not there was $12.88 \mathrm{sec}$ for the semantic-search condition and $14.67 \mathrm{sec}$ for the letter-search condition $[F(1,47)=$ $23.95, p<.01\}$. In general, then, the latencies were consistently longer for the letter-search condition, and the disadvantage for that condition increased as the target appeared later in the story.

\section{Discussion}

Contrary to expectation from the pilot data, the absolute magnitudes of the effects obtained in Experiment 2 were not attenuated in comparison with those obtained in Experiment 1. In fact, subsequent subanalyses of the data from the first two experiments indicated that almost all the effects were unambiguously clear after the first 12 to 18 subjects.

In terms of specific outcomes, the data indicate that the subjects were able to find a target word within the stories faster when they were searching for a word with a particular meaning than when they were searching for a word with a particular first letter; the later the target appeared in the story, the longer it took to find it; and, finally, the reliable interaction between target type and target position would suggest that with increased reading time the expected magnification of the effect of target type did occur. As in the first experiment, then, the data seem to suggest that during reading, the semantic representation of words is more immediately available for use as a basis for determining a response than is the specific component-letter information. This would seem to offer support for the type of position characterized by the pattern-unit model.

\section{EXPERIMENT 3}

The last question examined in this series of experiments concerned whether there were any differences between a task in which the subjects were to search for an item that belonged to a particular semantic category and a task in which they were to search for a specific predesignated word. Basically, the question resolves to asking whether one can complete a semantic analysis of a word as fast as one can detect that a predesignated word has appeared.

If one were to assume a view similar to that proposed by Massaro and Klitzke (1977), the task of detecting a predesignated target word might be expected to involve less processing than the task of determining the meaning of a word. That would be particularly true if the targets and foils did not have a large number of overlapping letters. Under those circumstances, the detection decision could be made immediately after the first feature had been extracted from each letter position, while a far more extensive encoding of a visual display would be required if the subject was to use the encoding as a basis for determining a meaning within semantic memory. This model, then, would seem to suggest that the time needed to detect a predesignated target word would be less than the time needed to find a word belonging to a predesignated target category.

The pattern-unit model, on the other hand, would suggest that such a difference in reaction time might not appear. That is, it is assumed that encodings of the components of a rule-governed pattern (e.g., a word) are never available to the subject in a form that would allow truncated processing. That would lead one to expect a very complete analysis of a word, regardless of the nature of the task (i.e., even including the case in which the task requires detecting only the first letter). Furthermore, given a standard reading situation, word processing may be more or less automatic up through a semantic encoding. That should mean that the time to detect a word would be about the same as the time needed to determine that it belonged to a particular semantic category, whereas a decision regarding a 
component letter would have to be delayed until after the word-level code had been decoded.

While the first two experiments, as well as the prior data (Johnson, 1975; Sloboda, 1976, 1977), demonstrate the type of delay expected when a subject must process a letter within a word, the present experiment was designed to examine the relative availability of the information used to detect a word as opposed to the information needed to identify the meaning of a presented word. The task involved the standard set of stories, and the subjects searched for either a specific word or a word that fit a specific semantic category.

\section{Method}

The methods, materials, and procedures for this experiment were identical to those used in the first experiment, with the single exception that the letter-search task was replaced by a task in which the predesignated target was a specific word (i.e., the target word). In the case of the "no" items, in which the targets did not appear in the story, the original targets had been selected in terms of specific words that were consistent with the stories, and those words were the items used as the targets for the word-search condition. The subjects were 24 introductory psychology students who participated as part of a course option.

\section{Results}

The latency results of the experiment are presented in Table 3, along with the error frequencies. There were only eight errors in the experiment (1.4\%), with five in the semantic-search condition and three in the wordsearch condition. The errors were distributed across eight subjects (one per subject), and while there were three errors for the middle portion of the semanticsearch condition, each of the other five conditions had only one error. Again, all the errors were failures to detect a target, and, as with Experiment 1, the low overall error rate and even distribution did not seem to warrant a statistical analysis.

The comprehension scores also were consistent with the data obtained in Experiment 1. For the word-search condition, the mean number of items correct was 14.75 , and it was 14.67 for the semantic-search condition $(\mathrm{F}<1.00)$. Therefore, in terms of accuracy during reading and both the comprehension and retention of the material, there appeared to be no evidence of a difference between the two search conditions.

An analysis of the latency results in Table 3 indicates

Table 3

Latency Results (in Seconds) from Experiment 3

\begin{tabular}{|c|c|c|c|c|c|c|c|c|}
\hline \multirow{3}{*}{$\begin{array}{l}\text { Type of } \\
\text { Search }\end{array}$} & \multicolumn{6}{|c|}{ Target Position } & & \\
\hline & \multicolumn{2}{|c|}{ Early } & \multicolumn{2}{|c|}{ Middle } & \multicolumn{2}{|c|}{ Late } & \multicolumn{2}{|c|}{ Mean } \\
\hline & RT & EF & RT & EF & RT & $\mathrm{EF}$ & RT & EF \\
\hline $\begin{array}{l}\text { Word } \\
\text { Semantic }\end{array}$ & $\begin{array}{l}1.60 \\
1.78\end{array}$ & $\begin{array}{l}1 \\
1\end{array}$ & $\begin{array}{l}6.48 \\
6.91\end{array}$ & $\begin{array}{l}1 \\
3\end{array}$ & $\begin{array}{l}11.02 \\
10.79\end{array}$ & $\begin{array}{l}1 \\
1\end{array}$ & $\begin{array}{l}6.36 \\
6.49\end{array}$ & $\begin{array}{l}3 \\
5\end{array}$ \\
\hline Mean & 1.69 & 2 & 6.70 & 4 & 10.91 & 2 & 6.43 & 8 \\
\hline
\end{tabular}

Note $-R T=$ reaction time, $E F=$ error frequency. a very marked effect of target position $[F(2,46)=$ $599.06, \mathrm{p}<.001]$, but neither the effect of the type of target $(F<1.00)$ nor the interaction $[F(2,46)=2.77$, $p>.05]$ was reliable. In addition, however, while the time to finish reading the stories when the target did appear was not different for the two search conditions (12.93 sec for semantic search and $12.55 \mathrm{sec}$ for word search) $[F(1,23)=1.69, p>.05]$, there was a marked advantage for the word-search condition for the stories in which the target did not appear (10.86 vs. $11.93 \mathrm{sec})$ $[F(1,23)=13.71, p<.001]$.

\section{Discussion}

With the exception of the time needed to indicate that the target had not appeared in the story, the results are consistent in indicating that the processing time involved in ascertaining that a presented word matches a predesignated target word is the same as the time needed to determine that the meaning of a presented word matches a predesignated target meaning. That would seem to suggest that the two pieces of information from the display were equally available for processing at the time of the comparison with the memorial representation of the target, and it may be that word processing proceeds in an automatic manner (LaBerge \& Samuels, 1974) through a semantic encoding.

The fact that the subjects were slower in making a negative response when the target had been specified in terms of a semantic category is not all that surprising. In that the categories used were rather clearly defined and the category instances were unambiguous, the subjects should have had no trouble identifying a target when it appeared, and the data seem to bear out that point. However, in the event that a target did not appear, the only issue is the clarity of the target specification, and it seems obvious that from the subject's point of view the specification is considerably greater if he is told to look for the word "blanket" than if he is told to look for "the name of an item commonly found on a bed." Given that is the case, the subjects should have been much more certain that the story did not contain the target if they had failed to find a specific word than if they had failed to find a word belonging to a particular semantic category. That differential certainty could account for the results.

\section{GENERAL DISCUSSION}

The overall issue addressed in this series of experiments was not the traditional question of whether one "sees" the components of a pattern before "seeing" the whole pattern. A serious analysis of that question forced us to conclude that there must be some sense in which perceptual representations of the components of a pattern are available either before or at the same time as a representation of the pattern as a whole. An experiment did not seem to be necessary to answer that question. 
However, it was suggested that a more meaningful question might concern the form in which the information is available, with the specific issue being the availability of the type of postperceptual cognitive encoding needed to determine a response. In particular, it was noted that one type of model does not assume the availability of such a code for a pattern's components prior to the availability of such code for the pattern itself. In addition, one specific model of that type (i.e., the pattern-unit model) takes the rather extreme view that such encodings for the components are never available prior to an encoding for the pattern as a whole and can be recovered only by decoding the pattern-level code after it has been established. It was to this particular question that the experiments were addressed in an effort to examine the utility of the pattern-unit model.

\section{The Data}

In terms of this issue, the results of the three experiments seem relatively clear. The time needed to determine that a specific word had appeared in a story was about the same as the time needed to determine that a word with a specific meaning had appeared, and the latter determination was made in substantially less time than was needed to indicate that a word began with a predesignated target letter.

The argument that the delay in responding to a letter within a word can be attributed to a lateral masking effect is contraindicated by two important considerations. First, if the subjects have no prior knowledge as to the specific word for which they are searching, they need rather complete information from each of the letter positions in order to make a decision, and processing should be maximally affected by any possible lateral inhibition effects. However, when the semanticsearch condition was compared with a condition in which the subjects were asked to process only the first letter of the word (i.e., the single letter position least susceptible to lateral inhibition), the word-level advantage still occurred. Given that, the credibility of the lateral inhibition interpretation is severely reduced.

More important to the issue of lateral inhibition, however, is the previously described work of both Sloboda (1976, 1977) and Johnson and Pettegrew (Note 1), which indicates that a word advantage is obtained even when the requisite stimulus information is exactly the same for a word-search and a letter-search condition. There seems to be no way that differential lateral inhibition can handle that effect. That is, the specific stimulus information to which the subject would have to respond would be exactly the same, and if lateral inhibition reduced its clarity, there would have to be an equal reduction of the critical stimulus information for both conditions.

\section{The Massaro and Klitzke Model}

Regarding the Massaro and Klitzke (1977) position, the type of advantage they describe for word processing over letter processing seems to be dependent upon knowing the target item in advance. That is, to be able to respond to a displayed word the instant you know that the first feature extracted from each letter position either is or is not consistent with the target requires foreknowledge of the visual characteristics of the target. In the present experiments, however, an advantage for word-level processing was obtained even when the sub. ject did not have prior information regarding the visual characteristics of the target words, and that would seem to be a difficult point for their model to handle.

In the absence of foreknowledge regarding the visual characteristics of the target, Massaro and Klitzke (1977) suppose that both word and letter identification proceed as two-stage processes. Regardless of whether a display to be analyzed is a letter in isolation or the subject is to identify a letter within a word, the first stage of letter identification would be to determine the general shape or envelope of the letter. In that there are many letters that share the same envelope, it would be necessary to proceed further to the second stage of processing, which "resolves the details of the envelope of the letter" (Massaro \& Klitzke, 1977, p. 296).

In the case of a word, on the other hand, Massaro and Klitzke (1977) assume that the first stage of processing also is the identification of an envelope for each letter but that the second stage of detail resolution is unnecessary. In place of the second stage, they assume that subjects identify all the letters that are consistent with the envelope for each letter position and then generate all possible combinations of these letters for consideration. The rules of English orthography and meaning are then applied to select a set of candidates that are acceptable in the language. If the selected set exceeds one, which surely must be the case for a large portion of possible words, subjects have to apply some unspecified procedure to select the one possibility that is correct.

In order to account for why subjects might do better on word identification than on letter identification, Massaro and Klitzke (1977) assume that the foregoing account of the second stage of word identification takes less time than does the second stage of letter processing, which involves nothing more than resolving the details of the envelope of one letter. While there may be no direct evidence to disconfirm this assumption, it does strain the bounds of credibility to the point where it is difficult to consider the model as a serious candidate for explaining the process of word identification. In addition, however, the fact that subjects can respond to the meaning of a displayed word just as fast as they can determine that it matches a predesignated target word also raises problems for this type of position. To this extent, then, the patternunit model does seem to be a somewhat more viable possibility.

In summary, these experiments seem to suggest that the type of information subjects need in order to make decisions regarding the components of a small pattern is not as immediately available as is the pattern-level 
information. Furthermore, Experiment 3 would suggest that the immediately available pattern-level information is rather complete, in that subjects can make decisions regarding the semantic characteristics of a display with as much ease as they can detect the appearance of a predesignated target word. In general, then the data seem quite consistent with a model of visual information processing that assumes that a cognitive encoding of a word need not be preceded by an analogous encoding of its components. Indeed, the results appear to indicate that such a prior cognitive encoding of the components cannot occur.

\section{REFERENCE NOTE}

1. Johnson, N. F., \& Pettegrew, B. Part-whole relationships in the processing of small visual arrays. Unpublished manuscript, 1979. (Available from N. F. Johnson, Department of Psychology, Ohio State University, Columbus, Ohio 43210.)

\section{REFERENCES}

Biederman, I. Perceiving real-world scenes. Science, 1972, 177, 77-80.

Cattell, J. M. The time taken up by cerebral operations. Mind, 1886, 11, 377-392.

Henderson, L. Do words conceal their component letters? A critique of Johnson (1975) on the visual perception of words. Journal of Verbal Learning and Verbal Behavior, 1975, 14, 648-650.

Johnson, N. F. On the function of letters in word identification: Some data and a preliminary model. Journal of Verbal Learning and Verbal Behavior, 1975, 14, 17-29.

Johnson, N. F. A pattern-unit model of word identification. In D. LaBerge \& S. J. Samuels (Eds.), Basic processes in reading: Perception and comprehension. Hillsdale, N.J: Erlbaum, 1977.

LaBerge, D. Perceptual learning and attention. In W. Estes (Ed.), Handbook of learning and cognitive processes (Vol. 4). Hillsdale, N.J: Erlbaum, 1976.

LaBerge, D., \& SAmuels, S. J. Toward a theory of automatic information processing in reading. Cognitive Psychology, 1974, 6, 293-323.

Marmurek, H. H. C. Processing letters in words at different levels. Memory \& Cognition, 1977, 5, 67-72.

Massaro, D. W., \& Klitzke, D. Letters are functional in word identification. Memory \& Cognition, 1977, 5, 292-298.

Navon, D. Forest before trees: The precedence of global features in visual perception. Cognitive Psychology, 1977, 9, 353-383.

Palmer, S. E. Hierarchical structure in perceptual representation. Cognitive Psychology, 1977, 9, 441-474.

Stoboda, J. A. Decision times for word and letter search: A wholistic word identification model examined. Journal of Verbal Learning and Verbal Behavior, 1976, 15, 93-101.

Sloвoda, J. A. The locus of the word-priority effect in a targetdetection task. Memory \& Cognition, 1977, 5, 371-376.

(Revision accepted for publication August 23, 1979.) 